\title{
Lower Bounds on the Success Probability for ad hoc Networks with local FDMA scheduling
}

\author{
Ralph Tanbourgi, Jens P. Elsner, Holger Jäkel and Friedrich K. Jondral \\ Karlsruhe Institute of Technology, Germany \\ $\{$ ralph.tanbourgi, jens.elsner, holger.jaekel, friedrich.jondral $\} @$ kit.edu
}

\begin{abstract}
This paper studies the performance of ad hoc networks with local FDMA scheduling using stochastic point processes. In such networks, the Poisson assumption is not justified due to interdependencies between points introduced by scheduling. For this reason, an upper bound on the second reduced moment measure is derived. Using this result, two lower bounds on the success probability are given, based on the second order product density and a non-homogeneous Poisson approximation. The relative performance of local FDMA is compared to random channel access. It is shown that the relative outage probability reduction of local FDMA highly depends on the SIR threshold as well as on the ratio of transmission distance to orthogonalization distance. If these two quantities are small, the improvement is high; the number of channels has only a minor effect on the relative improvement.
\end{abstract}

\section{INTRODUCTION}

The fundamental limits of general wireless networks in terms of capacity are not fully understood yet and extensive research in this area is still ongoing. A well-accepted opinion, however, is that co-channel interference is the performancelimiting factor in (dense) wireless networks [1], [2]. In order to properly capture the impact of the uncertainty in the spatial configuration on the performance of the network, tools from stochastic geometry, most notably the theory of Poisson point processes, have been successfully applied to uncoordinated wireless networks (see [3], [4]).

The investigation of wireless networks employing coordinated medium access to yield better medium utilization in this framework is challenging, since the Poisson assumption is no longer justified. Recent works study the effect of transmitter clustering [5] and carrier sense multiple access (CSMA) [4]. The work on CSMA has been extended by taking into account performance guarantees [6] and nodes with cognitive abilities [7].

Another promising approach for achieving coordination among the nodes is local FDMA scheduling. Here, a wireless multi-channel network is considered, in which the nodes are able to locally coordinate their channel assignments. The benefits of local scheduling have been studied in [8], [9] for different types of scheduling. In [8], the success probability and transmission capacity of a local FDMA based ad hoc network was analyzed for the path loss model. In particular, the question of network wide orthogonalization has been addressed. Nearest neighbor FDMA scheduling has been studied in [9], where it was assumed that the number of orthogonal channels is sufficiently large such that every receiver can orthogonalize its nearest interferer. In these two works, results have been derived and validated by simulations without characterizing the resulting point process in detail.

It is straightforward to see that the underlying point process of such scheduled networks is not Poisson, since the scheduling component creates interdependencies between the nodes. Unfortunately, the moment-generating functional for non-Poisson point processes are not known in closed form and one has to resort to approximations. For instance, in [4] the success probability for CSMA is derived by approximating the characteristics of the underlying Matern hard-core process by a non-homogeneous Poisson point process. Another way of calculating the success probability and transmission capacity was suggested in [10], where the key idea is to use product densities, which are known for several point processes.

This work aims at investigating the interference situation in a wireless ad hoc network, in which nodes are capable of jointly and locally orthogonalizing their transmission schedules using orthogonal channels. We derive an upper bound on the second reduced moment measure and the second order product density. These results are then used to bound the success probability of the scheduled network in order to show the gain of local FDMA scheduling. In particular, we analyze the relative improvement of FDMA compared to unscheduled random channel access in terms of outage probability for low node intensities.

\section{SySTEM MODEL}

\section{A. Network Geometry}

Let $\left\{X_{i}\right\}$ be the set of transmitters which are uniformly and independently distributed in the plane $\mathbb{R}^{2}$ according to a stationary Poisson point process (PPP) $\Phi$. The intensity of $\Phi$ is given by $\lambda$. We define $\Phi$ as a random set, i.e.,

$$
\Phi(A) \triangleq\left\{X_{i} \mid X_{i} \in A\right\}, \quad A \subseteq \mathbb{R}^{2} .
$$

Each transmitter $X_{i}$ has an intended receiver $X_{i}^{\mathrm{rx}}$ randomly situated at most $r$ units away, where the actual positions of the $X_{i}^{\mathrm{rx}}$ are uniformly distributed within $b\left(X_{i}, r\right)$. The set of receivers $\left\{X_{i}^{\mathrm{rx}}\right\}$ forms another stationary PPP denoted by $\Phi^{\mathrm{rx}}$. Slivnyak's Theorem [11] states that the distribution of $\Phi$ is not changed by the addition of a point (without counting it). Consequently, we can place a reference ${ }^{1}$ receiver $X_{0}^{\mathrm{rx}}$ in the

\footnotetext{
${ }^{1}$ We index all reference quantities by " 0 ". Consequently, $i \in \mathbb{N}^{+}$for both $\Phi$ and $\Phi^{\mathrm{rx}}$.
} 
origin and a reference transmitter $X_{0}$ separated by $d_{0}$ units, where $d_{0} \leq r$. The interference situation will be considered from the viewpoint of $X_{0}^{\mathrm{rx}}$.

We assume that the total available bandwidth is equally split into $M$ orthogonal channels $m \in \mathbb{M}=\{1, \ldots M\}$. At the time of medium access, each pair $X_{i}, X_{i}^{\mathrm{rx}}$ (including the reference pair) has chosen a certain channel $m_{i}$. Hence, we can consider the collection of tuples $\left\{\left(X_{i}, m_{i}\right)\right\}$ (and also $\left\{\left(X_{i}^{\text {rx }}, m_{i}\right)\right\}$ ) defined on the Cartesian product $\mathbb{R}^{2} \times \mathbb{M}$ and define the marked point process $\tilde{\Phi}$ (and $\tilde{\Phi}^{\mathrm{rx}}$ ) as

$\tilde{\Phi}(A \times B)=\left\{\left(X_{i}, m_{i}\right) \mid X_{i} \in A, m_{i} \in B\right\}, A \subseteq \mathbb{R}^{2}, B \subseteq \mathbb{M}$.

We denote by $m_{0}$ the reference mark, i.e., the mark associated with the reference pair $X_{0}, X_{0}^{\mathrm{rx}}$.

\section{B. Local FDMA Scheduling}

Local FDMA scheduling aims at coordinating channel access among co-located nodes in order to avoid excessive co-channel interference. Similarly as in CSMA/CA where a ready-to-send/clear-to-send (RTS/CTS) exchange is necessary prior to transmission, local FDMA scheduling requires a contention resolution period in which the channels are allocated through local signaling, e.g., by sending beacon signals to indicate the chosen channel. During this contention resolution period, co-located nodes jointly assign their channels in such a way that no receiver $X_{i}^{\text {rx }}$ has an interferer $X_{j}$ within its proximity transmitting in the same channel. Proximity is defined in the Euclidean sense by a disc $b(x, r)$ of radius $r$ around a point $x$.

We use the concept of conflict graphs to formalize the scheduling problem. A transmission pair, indicated by the tuple $\left(X_{i}, X_{i}^{\mathrm{rx}}\right)$ is said to be in conflict with another pair $\left(X_{j}, X_{j}^{\mathrm{rx}}\right)$, if either $X_{i} \in b\left(X_{j}^{\mathrm{rx}}, r\right)$ or $X_{j} \in b\left(X_{i}^{\mathrm{rx}}, r\right)$. Considering the tuples $\left(X_{i}, X_{i}^{\mathrm{rx}}\right)$ as vertices, a conflict graph can be constructed with edges connecting those pairs creating a conflict to each other. We denote by $C_{i}$ the set of conflicting nodes associated with the transmission pair $\left(X_{i}, X_{i}^{\mathrm{rx}}\right)$, i.e.,

$$
\begin{aligned}
C_{i}=\Phi( & \left(b\left(X_{i}^{\mathrm{rx}}, r\right)\right) \backslash X_{i} \\
& \cup\left\{X_{j}^{\mathrm{rx}} \in \Phi^{\mathrm{rx}}\left(b\left(X_{i}, r\right)\right) \mid X_{j} \notin b\left(X_{i}^{\mathrm{rx}}, r\right)\right\},
\end{aligned}
$$

Note that the additional property $X_{j} \notin b\left(X_{i}^{\mathrm{rx}}, r\right)$ in the second set avoids counting conflicts twice.

From the theorem of Brooks [12] it follows that every graph can be colored properly ${ }^{2}$ with at $\operatorname{most} \max _{i}\left\{\left|C_{i}\right|\right\}+1$ colors, where $\left|C_{i}\right|$ is the cardinality of $C_{i}$. Unfortunately, in our network model, there is a non-zero probability of an unbounded vertex degree due to the fact that infinitely many points may be close to each other. Hence, we cannot determine the minimum number of colors required for proper coloring. Furthermore, in practice the number of channels is typically given by the system design constraints (e.g., spectrum regulation, hardware complexity, desired data rate). As a result, the dependently marked point process $\tilde{\Phi}$ may not fulfill the

\footnotetext{
${ }^{2} \mathrm{~A}$ coloring is proper if no two vertices connected by an edge have the same color.
}

scheduling policy at all nodes due to an insufficient number of channels (non-proper coloring).

In the following, we consider $\tilde{\Phi}$ (and $\tilde{\Phi}^{\mathrm{rx}}$ ) at the time the scheduling task has terminated. ${ }^{3}$ Furthermore, only the transmitters with marks equal to the reference mark $m_{0}$ will be considered, since only these will contribute to the aggregated interference. The collection of these interferers is denoted by $\tilde{\Phi}_{0}$, i.e.,

$$
\tilde{\Phi}_{0}(A) \triangleq \tilde{\Phi}\left(A \times m_{0}\right)=\left\{\left(X_{i}, m_{i}\right) \mid X_{i} \in A, m_{i}=m_{0}\right\},
$$

which is not Poisson but stationary [4]. The intensity of $\tilde{\Phi}_{0}$ is equal to $\frac{\lambda}{M}$ due to symmetry reasons, i.e., no specific channel is preferred by the scheduling algorithm.

\section{Second Reduced Moment Measure of $\tilde{\Phi}_{0}$}

The second reduced moment measure $\mathcal{K}_{2}$ of a point process is defined as

$$
\mathcal{K}_{2}(b(0, t))=\frac{M}{\lambda} \mathbb{E}^{! o}\left\{\sum_{i} \mathbb{1}_{\left\{X_{i} \in \tilde{\Phi}_{0}(b(0, t))\right\}}\right\} .
$$

This means we count the number of points from $\tilde{\Phi}_{0}$ that fall into $b(0, t)$ without counting the reference point in the origin and normalize this quantity by the intensity $\lambda / M$. In the isotropic case, (5) can be written in terms of the second reduced moment function $K(t)=\mathcal{K}_{2}(b(0, t))$. We now propose an upper bound on $K(t)$ which will be used for the analysis of the success probability later on.

\section{A. Upper Bound on the Second Reduced Moment Measure}

Theorem 1. The expected number of interferers within a disc of radius $t$ around a receiver $X_{0}^{\mathrm{rx}}$ located in the origin, given a stationary point process $\tilde{\Phi}$ with marks assigned according to the scheduling policy of (3), is upper bounded by

$$
\begin{aligned}
& \frac{\lambda}{M} K(t)=\mathbb{E}^{! o}\left\{\sum_{i} \mathbb{1}_{\left\{X_{i} \in \tilde{\Phi}_{0}(b(0, t))\right\}}\right\} \\
& \leq \frac{\lambda}{M} K^{\mathrm{u}}(t)=\left\{\begin{array}{l}
\Delta t^{2}, \quad t \leq r \\
\min \left\{\Delta r^{2}+\lambda \pi\left(t^{2}-r^{2}\right), \frac{\lambda}{M} \pi t^{2}\right\}, t>r,
\end{array}\right.
\end{aligned}
$$

where

$$
\Delta=\frac{e^{-\left(2 \lambda \pi r^{2}-\zeta\right)}}{2 M r^{2}} \sum_{k=M}^{\infty}(k-M+1)\left(\lambda \pi r^{2}\right)^{k} \sum_{\ell=0}^{k} \frac{\left(1-\frac{\zeta}{\lambda \pi r^{2}}\right)^{k-\ell}}{\ell !(k-\ell) !}
$$

and

$\zeta=\frac{2 \lambda}{\pi r^{2}} \int_{0}^{r+d} z \Omega(z)\left[2 r^{2} \arccos \left(\frac{z}{2 r}\right)-\frac{z}{2} \sqrt{4 r^{2}-z^{2}}\right] \mathrm{d} z$,

where

$\Omega(z)= \begin{cases}\pi, & 0 \leq r \leq r-d \\ \arccos \left(\frac{r^{2}-d^{2}-z^{2}}{-2 d z}\right), & r-d<z \leq r+d .\end{cases}$

Proof: The proof is given in appendix A.

${ }^{3}$ Either by having found a proper coloring or by aborting after a predefined number of iterations. 


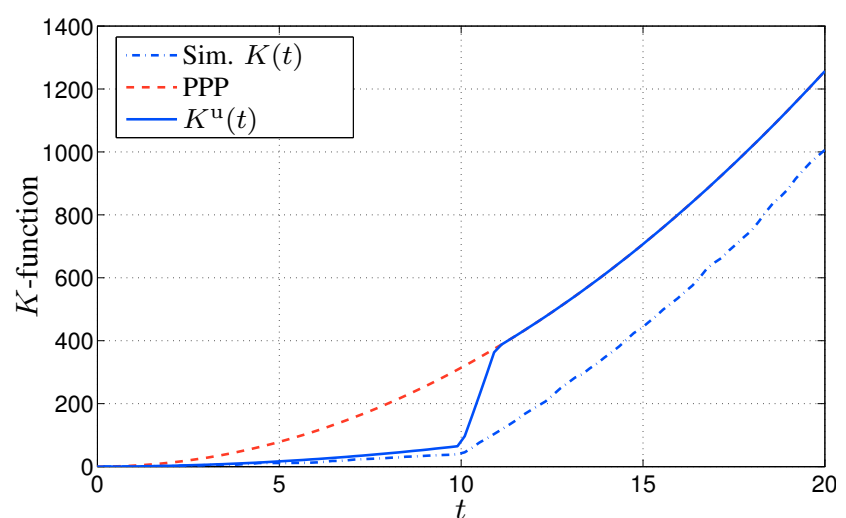

Fig. 1. The simulated $K(t)$ and the corresponding upper bound $K^{\mathrm{u}}$ with parameters $\lambda=10^{-2}, M=5, d=7$ and $r=10$.

Fig. 1 shows the upper bound $K^{\mathrm{u}}(t)$ together with the simulated $K(t)$ and the homogeneous Poisson case $\pi t^{2}$. We have used a decentralized stochastic coloring algorithm, namely the communication-free learning (CFL) algorithm from [13], for implementing the scheduling policy. One can observe that $K(t)$ increases very slowly until the scheduling range $t=r$ is reached. The bound $K^{\mathrm{u}}(t)$ performs well in the region $t \leq r$. For sufficiently large $M$, the slope of $K(t)$ tends to zero in this region and $\tilde{\Phi}_{0}$ becomes comparable to a hard-core point process.

\section{B. Upper Bound on the Second Order Product Density}

From [11], we have the following relation

$$
\rho^{(2)}(t)=\frac{\lambda^{2}}{2 \pi t M^{2}} \frac{\partial K(t)}{\partial t},
$$

where $\rho^{(2)}(t)$ is the second order product density of a stationary point process (on $\mathbb{R}^{2}$ ) with intensity $\lambda / M$.

We would like to know if the upper bound $K^{\mathrm{u}}(t)$ from (6) results in an upper bound on $\rho^{(2)}$ after differentiation. We first analyze the case $t \leq r$ : It is reasonable to assume that the location of a non-scheduled interferer is uniformly distributed within $b(0, r)$ since there is no reason for a spatial orientation of non-scheduled interferers. Consequently, the derivate of $K(t)$ grows linearly. Since $K^{\mathrm{u}}(0)=K(0)=0$ and $K^{\mathrm{u}}(t) \geq K(t)$ it follows that $\partial K^{\mathrm{u}}(t) / \partial t=c \partial K(t) / \partial t$ with $c \geq 1, \forall t \in[0, r]$.

For $t>r, K^{\mathrm{u}}(t)$ grows certainly faster than $K(t)$ due to the fact that the upper bound was constructed by assuming all interferers transmit in the reference channel $m_{0}$. Hence, combining (6) and (7) yields the following upper bound on $\rho^{(2)}(t)$

$$
\rho^{(2)}(t) \leq \rho^{(2), \mathrm{u}}(r)= \begin{cases}\frac{\lambda}{\pi M} \Delta & t \leq r \\ \frac{\lambda^{2}}{M} & r<t<t_{0} \\ \frac{\lambda^{2}}{M^{2}} & t>t_{0},\end{cases}
$$

where

$$
t_{0}=r \sqrt{\frac{\lambda \pi-\Delta}{\lambda \pi(1-1 / M)}}
$$

is the intersection point of $\Delta r^{2}+\lambda \pi\left(t^{2}-r^{2}\right)=\lambda \pi t^{2} / M$.

\section{LOWER Bounds on the SuCCESS PROBABILITY}

We define the following quantities:

- $g_{0}$ and $\left\{g_{i}\right\}$ : Unit-mean exponentially distributed channel gains from $X_{0}$ to $X_{0}^{\mathrm{rx}}$ and from all interferers $X_{i}$ to $X_{0}^{\mathrm{rx}}$, respectively.

- $d_{i}$ : Distance from transmitter $X_{i}$ to reference receiver $X_{0}^{\mathrm{rx}}$, i.e., $d_{i} \triangleq\left|X_{i}-X_{0}^{\mathrm{rx}}\right|=\left|X_{i}\right|$.

- $\ell(x)=|x|^{-\alpha}$ : Path loss function ${ }^{4}$ with path loss exponent $\alpha>2$.

- $\beta$ : Required signal-to-interference (SIR) ratio.

- $I_{0}=\sum_{X_{i} \in \tilde{\Phi}_{0}} g_{i} \ell\left(d_{i}\right)$ : Aggregated interference in the reference channel $m_{0}$ measured at the reference receiver $X_{0}^{\mathrm{rx}}$ without counting the reference transmitter $X_{0}$.

The probability of success is defined as the probability of the SIR being higher than a certain threshold $\beta$ [14], i.e.,

$$
p_{s} \triangleq \mathbb{P}^{! o}\left\{\frac{g_{0} d_{0}^{-\alpha}}{I_{0}} \geq \beta\right\} .
$$

This can be expressed as [10]

$$
p_{s}=\mathbb{P}^{! o}\left\{g_{0} \geq \beta d_{0}^{\alpha} I_{0}\right\}=\mathbb{E}^{! o}\left\{\prod_{i} 1-v\left(d_{i}\right)\right\},
$$

where

$$
v(d)=\frac{1}{1+\beta^{-1} d_{0}^{-\alpha} d^{\alpha}} .
$$

\section{A. Lower Bound: Second Order Product Density Method}

In recently published work [10], a lower and upper bound on $p_{s}$ was proposed which is based on the second- and third order product densities. For the lower bound, the authors make use of the relation

$$
\mathbb{E}^{! o}\left\{\sum_{i} f\left(X_{i}\right)\right\}=\lambda^{-1} \int_{\mathbb{R}^{2}} \rho^{(2)}(x) f(x) \mathrm{d} x
$$

for a point process of intensity $\lambda$ to obtain [10, Lemma 2]

$$
p_{s} \geq 1-\lambda^{-1} \int_{\mathbb{R}^{2}} \rho^{(2)}(x) v(x) \mathrm{d} x .
$$

From combining (8), (12) and (14), it follows that

$$
\begin{aligned}
p_{s} & \geq p_{s}^{1} \\
& \triangleq 1-2 \Delta \int_{0}^{r} t v(t) \mathrm{d} t-2 \pi \lambda \int_{r}^{t_{0}} t v(t) \mathrm{d} t-2 \pi \frac{\lambda}{M} \int_{t_{0}}^{\infty} t v(t) \mathrm{d} t .
\end{aligned}
$$

\footnotetext{
${ }^{4}$ Other path loss functions resolving the singularity problem at $d=0$ can also be used instead.
} 


\section{B. Lower Bound: Non-homogeneous Poisson Approximation}

In this approach, the characteristics of $I_{0}$, conditioned on the fact that $X_{0}^{\mathrm{rx}}$ with mark $m_{0}$ lies in the origin, is approximated by a non-homogeneous PPP with intensity $\lambda(t)=\lambda / M \partial K / \partial t$. A similar approach was performed in [4] to analyze the success probability for CSMA. Using the Laplace functional of a PPP (cf. [4], [11]), we can thus approximate the lower bound on $p_{s}$ according to

$$
p_{s} \gtrsim p_{s}^{\mathrm{l} \text {,ap }} \triangleq \exp \left\{-\frac{\lambda}{M} \int_{\mathbb{R}^{+}} \frac{\partial K^{\mathrm{u}}(t)}{\partial t} v(t) \mathrm{d} t\right\} .
$$

Remark: The right hand side of (15) may become greater than one as $\lambda$ increases. This bound is hence useful only for small $\lambda$ as already reported in [10]. For $\lambda \rightarrow 0$, (14) holds with equality and $p_{s}^{\mathrm{l}}-p_{s}^{\mathrm{l} \text {,ap }} \rightarrow 0$.

\section{Relative improvement of local FDMA}

For small intensities $\lambda$, the improvement of the proposed local FDMA compared to unscheduled random channel access ${ }^{5}$ can be easily analyzed using the relation $1-e^{-A} \approx A$ for $A \rightarrow 0$. The improvement $\eta$ is measured in terms of relative outage probability reduction when switching from unscheduled random channel access to locally scheduled FDMA, according to

$$
\eta \triangleq 1-\lim _{\lambda \rightarrow 0} \frac{1-p_{s, \text { sched }}}{1-p_{s, \text { unsched }}} .
$$

The success probability for the unscheduled case with unitmean exponentially distributed fading gains is given in [15] by $p_{s, \text { unsched }}=\exp \left\{-\frac{\lambda}{M} \pi\left(d^{\alpha} \beta\right)^{2 / \alpha} \Gamma(1-2 / \alpha) \Gamma(1+2 / \alpha)\right\}$. Since we only have a lower bound on $p_{s, \text { sched }}$ in the case of local FDMA, we will derive a lower bound on $\eta$, i.e., the minimum relative outage probability reduction, using (15). By inserting (15) in (17), evaluating the integrals and taking the limit, $\eta$ is lower bounded by

$$
\begin{aligned}
& \eta \geq \eta^{1} \\
& \triangleq 1-\lim _{\lambda \rightarrow 0} \frac{1-p_{s, \text { sched }}^{1}}{1-p_{s, \text { unsched }}} \\
&=\left(\gamma^{2} \beta^{-\frac{2}{\alpha}}{ }_{2} F_{1}\left(1, \frac{2}{\alpha} ; 1+\frac{2}{\alpha} ;-\beta^{-1} \gamma^{\alpha}\left(1-\frac{1}{M}\right)^{-\frac{\alpha}{2}}\right)\right. \\
&\left.\quad-\gamma^{2} \beta^{-\frac{2}{\alpha}}{ }_{2} F_{1}\left(1, \frac{2}{\alpha} ; 1+\frac{2}{\alpha} ;-\beta^{-1} \gamma^{\alpha}\right)+\frac{2 \pi}{\alpha M} \csc \left(\frac{2 \pi}{\alpha}\right)\right) \\
& \quad /\left(\frac{1}{M} \Gamma\left(1-\frac{2}{\alpha}\right) \Gamma\left(1+\frac{2}{\alpha}\right)\right),
\end{aligned}
$$

where $\gamma \triangleq \frac{r}{d_{0}}$ and ${ }_{2} F_{1}(a, b ; c ; z)$ is the hypergeometric function [16].

Fig. 2 shows the lower bound $\eta^{1}$ vs. the SIR threshold $\beta$ for different $\gamma$ and $M$. It can be seen that the relative reduction highly depends on the SIR threshold $\beta$ as well as on the ratio $\gamma$. Furthermore, the relative reduction is maximal for $M=2$ and decreases with $M{ }^{6}$ For $M \rightarrow \infty$,

\footnotetext{
${ }^{5}$ For instance, frequency hopping code division multiple access (FHCDMA).

${ }^{6}$ The absolute reduction of outage probability of course depends on the choice of $M$, cf. [8].
}

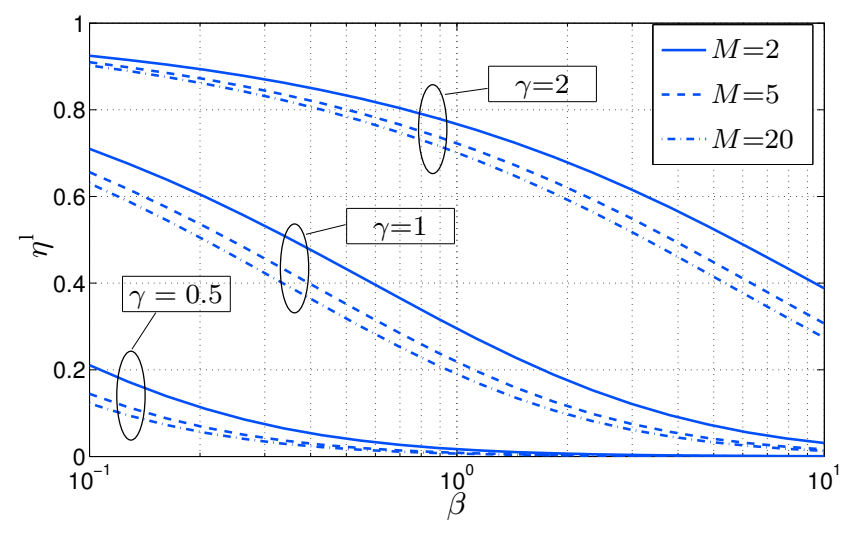

Fig. 2. Lower bound $(\lambda \rightarrow 0) \eta^{1}$ for the outage probability reduction vs. $\beta$ for different $M$ and $\gamma$.

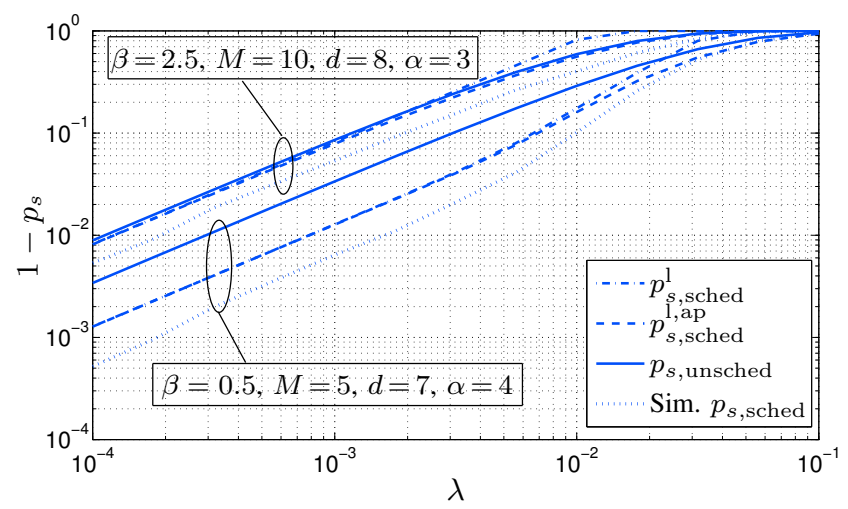

Fig. 3. Outage probability $1-p_{s}$ vs. density $\lambda$ for different $M, d, \beta, \alpha$, and $r=10$.

$\eta^{1} \rightarrow \frac{2}{\alpha} \pi \csc (2 \pi / \alpha)(\Gamma(1-2 / \alpha) \Gamma(1+2 / \alpha))^{-1}>0$. This is an interesting observation, since it states that there is always an improvement when switching to local FDMA, although the interference avoidance effect of unscheduled random channel access increases with $M$.

\section{Simulation Results}

Fig. 3 shows the outage probability $1-p_{s}$ vs. the node density $\lambda$ for uncoordinated random channel access and scheduled FDMA. The simulation results based on the CFL-algorithm as well as the analytical results (15) and (16), now representing upper bounds on the outage probability, are shown.

It can be seen that the bound $p_{s}^{1}$ is not very tight for high $\lambda$. This is a result of upper bounding the second order product density in (8) and of (14). The gain of local FDMA compared to uncoordinated random channel access is however noticeable, since the upper bound $1-p_{s}^{\mathrm{l}, \text { ap }}$ is always below the exact outage probabilities of unscheduled random channel access. Moreover, the simulation results reveal a significant reduction of outage probability compared to the unscheduled case.

The minimum relative outage probability reduction can be well observed comparing Fig. 2 and Fig. 3. Furthermore, the asymptotic behavior of $\eta^{1}$ already applies to node densities between $10^{-3}-10^{-2}$. 


\section{CONCLUSION}

As we have seen, local FDMA scheduling provides a significant advantage over uncoordinated channel access especially at low spectral efficiencies. This is an indicator in favor of spread spectrum channel access in ad hoc networks, something already observed for uncoordinated channel access in [17].

We have also shown that the gain over uncoordinated channel access is high when only a small number of channels are available and is lower bounded by a constant for high number of channels; an argument in favor of the implementation of coordination in frequency hopping large ad hoc network that have a small to moderate number of channels available.

The applied approximation methods yield some insight into the structurally complicated mathematical model of the interference field arising from a non-Poisson point processes with spatially correlated marks. Especially the $K$-function approximation proved to be a valuable tool as it can readily be determined empirically and be verified analytically.

\section{APPENDIX A}

\section{PROOF OF THEOREM 1}

Bound for $t \leq r$ : We condition $\tilde{\Phi}_{0}(b(0, t))$ on the fact that the reference transmission pair $X_{0}, X_{0}^{\mathrm{rx}}$ has $\left|C_{0}\right|=k$ conflicts that have to be colored, i.e.,

$$
\begin{aligned}
& \mathbb{E}^{! o}\left\{\sum_{i} \mathbb{1}_{\left\{X_{i} \in \tilde{\Phi}_{0}(b(0, t))\right\}}\right\}= \\
& \sum_{k=M}^{\infty} \mathbb{E}^{! o}\left\{\sum_{i} \mathbb{1}_{\left\{X_{i} \in \tilde{\Phi}_{0}(b(0, t))\right\}}|| C_{0} \mid=k\right\} \mathbb{P}\left\{\left|C_{0}\right|=k\right\} .
\end{aligned}
$$

For $k<M$, all conflicts are resolved since the reference pair can choose one of the remaining non-occupied channels. We first focus on the term $\mathbb{P}\left\{\left|C_{0}\right|=k\right\}$ : Using the definition from (3), this term can be calculated as

$$
\begin{aligned}
& \mathbb{P}\left\{\left|C_{0}\right|=k\right\}=\mathbb{P}\{\underbrace{\sum_{i} \mathbb{1}_{\left\{X_{i} \in C_{0}\right\}}}_{\mathrm{A}_{1}}+\underbrace{\sum_{i} \mathbb{1}_{\left\{X_{i}^{\mathrm{rx}} \in C_{0}\right\}}}_{\mathrm{A}_{2}}=k\} \\
& =\mathbb{P}\left\{\bigcup_{\ell=0}^{k}\left[\mathrm{~A}_{1}=\ell\right] \wedge\left[\mathrm{A}_{2}=k-\ell\right]\right\} \\
& \stackrel{\text { (a) }}{=} \sum_{\ell=0}^{k} \mathbb{P}\left\{\left[\mathrm{A}_{1}=\ell\right] \wedge\left[\mathrm{A}_{2}=k-\ell\right]\right\} \\
& \stackrel{\text { (b) }}{=} \sum_{\ell=0}^{k} \mathbb{P}\left\{\mathrm{A}_{1}=\ell\right\} \mathbb{P}\left\{\mathrm{A}_{2}=k-\ell\right\},
\end{aligned}
$$

where (a) follows from the fact that the events are disjoint, and (b) is a result of the independence between the two sums $A_{1}$ and $A_{2}$. The first sum $A_{1}$ is Poisson distributed with intensity $\lambda \pi r^{2}$. From the random translation theorem [4] it follows that the second sum $\mathrm{A}_{2}$ is also Poisson distributed with intensity

$$
\Lambda\left(b\left(X_{0}, r\right)\right)=\lambda \int_{\mathbb{R}^{2}} g\left(x, b\left(X_{0}, r\right)\right) \mathrm{d} x,
$$

where the probability kernel $g\left(x, b\left(X_{0}, r\right)\right)$ is the probability of a point $x$ to be shifted into $b\left(X_{0}, r\right)$, i.e.,

$$
g\left(x, b\left(X_{0}, r\right)\right)=\mathbb{1}_{\{x \notin b(0, r)\}} \mathbb{P}\left\{y \in b\left(X_{0}, r\right) \mid x\right\} .
$$

Note that the indicator function is necessary to require that $g\left(x, b\left(X_{0}, r\right)\right)=0$ for $x \in b(0, r)$, i.e., points coming from $b(0, r)$ are not taken into account here as they are creating conflicts to the reference receiver $X_{0}^{\mathrm{rx}}$ and hence, are already captured by the first sum.

Since the locations of the receivers $X_{i}^{\mathrm{rx}}$ are uniformly distributed in a disc of radius $r$ around their corresponding transmitter $X_{i}$, the term $\mathbb{P}\left\{y \in b\left(X_{0}, r\right) \mid x\right\}$ is proportional to the intersection area of $b(y, r)$ and $b\left(X_{0}, r\right)$, and is given by

$$
\begin{aligned}
\mathbb{P}\left\{y \in b\left(X_{0}, r\right) \mid x\right\} & =\frac{\left|b(y, r) \cap b\left(X_{0}, r\right)\right|}{|b(y, r)|} \\
& =\frac{2 r^{2} \arccos \left(\frac{|y|}{2 r}\right)-\frac{|y|}{2} \sqrt{4 r^{2}-|y|^{2}}}{\pi r^{2}} .
\end{aligned}
$$

See Fig. 4 for an illustration. Using (A.4), we can rewrite (A.3) as

$$
\begin{aligned}
\Lambda\left(b\left(X_{0}, r\right)\right) & =\lambda \int_{\mathbb{R}^{2}} \mathbb{1}_{\{x \notin b(0, r)\}} \mathbb{P}\left\{y \in b\left(X_{0}, r\right) \mid x\right\} \mathrm{d} x \\
& =\lambda \int_{\mathbb{R}^{2} \backslash b(0, r)} \mathbb{P}\left\{y \in b\left(X_{0}, r\right) \mid x\right\} \mathrm{d} x \\
& =\lambda \pi r^{2}-\lambda \underbrace{\lambda \int_{b(0, r)} \mathbb{P}\left\{y \in b\left(X_{0}, r\right) \mid x\right\} \mathrm{d} x}_{\triangleq \zeta} .
\end{aligned}
$$

After switching to polar coordinates, the integral in (A.6) can be written as

$$
\zeta=2 \lambda \int_{0}^{r+d} \int_{0}^{\Omega(z)} z \mathbb{P}\left\{y \in b\left(X_{0}, r\right) \mid z, \phi\right\} \mathrm{d} \phi \mathrm{d} z,
$$

where $0 \leq z \leq r+d$ and $0 \leq \phi \leq \Omega(z)$ define the points within $b(0, r)$ from the viewpoint of $X_{0}$, see Fig. 4. The function $\Omega(z)$ denotes the length of the circle with radius $z$ around the point $X_{0}$ that is contained in $b(0, r)$ and is given by

$$
\Omega(z)= \begin{cases}\pi, & 0 \leq z \leq r-d \\ \arccos \left(\frac{r^{2}-d^{2}-z^{2}}{-2 d z}\right), & r-d<z \leq r+d .\end{cases}
$$

Since $\mathbb{P}\left\{y \in b\left(X_{0}, r\right) \mid z, \phi\right\}=\mathbb{P}\left\{y \in b\left(X_{0}, r\right) \mid z\right\}$, we can rewrite (A.7) as

$$
\zeta=\frac{2 \lambda}{\pi r^{2}} \int_{0}^{r+d} z \Omega(z)\left[2 r^{2} \arccos \left(\frac{z}{2 r}\right)-\frac{z}{2} \sqrt{4 r^{2}-z^{2}}\right] \mathrm{d} z .
$$

Combining (A.3)-(A.9), we can finally rewrite (A.2) as

$$
\mathbb{P}\left\{\left|C_{0}\right|=k\right\}=\left(\lambda \pi r^{2}\right)^{k} e^{-\left(2 \lambda \pi r^{2}-\zeta\right)} \sum_{\ell=0}^{k} \frac{\left(1-\frac{\zeta}{\lambda \pi r^{2}}\right)^{k-\ell}}{\ell !(k-\ell) !} .
$$




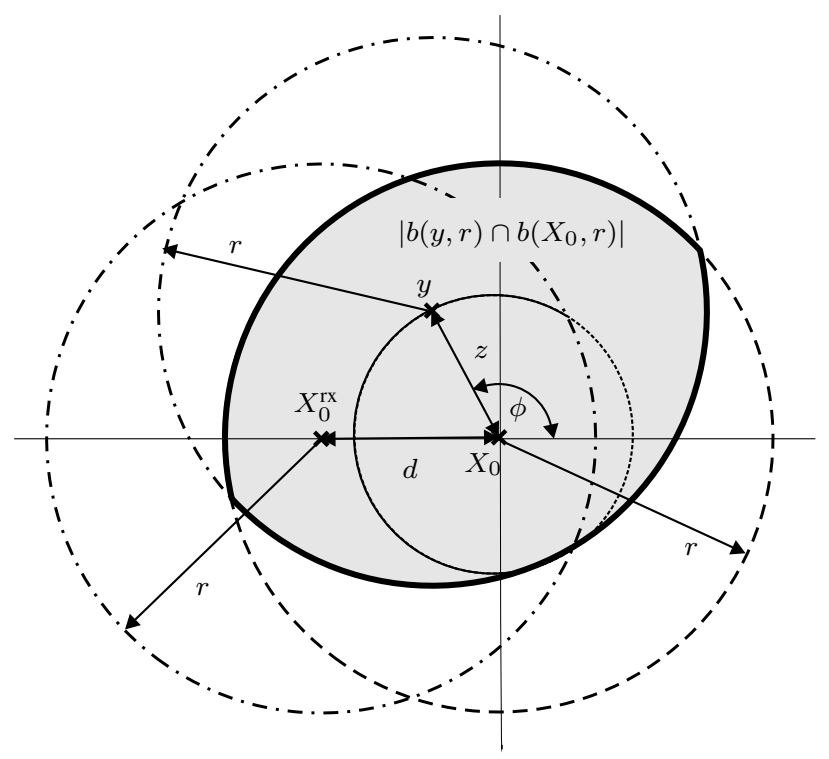

Fig. 4. Illustration supporting (A.5), (A.8) and (A.9).

Since the first $M-1$ conflicts are properly colored, we now consider the $k-M+1$ remaining conflicts. Without loss of generality, we index the nodes associated with these conflicts by $Y_{j}, j=1, \ldots, k-M+1$.

On average, $(k-M+1) / 2$ of these nodes will be transmitters. Thus we can state that $\mathbb{P}\left\{Y_{j}\right.$ is a transmitter $\}=1 / 2$.

Given $Y_{j}$ is a transmitter, it follows from the stationarity of $\Phi$ that this transmitter is located within $b(0, t)$ with probability $\mathbb{P}\left\{Y_{j} \in b(0, t) \mid Y_{j}\right.$ is transmitter $\}=t^{2} / r^{2}$. In order to interfere with $X_{0}^{\mathrm{rx}}$, this transmitter needs to transmit in the reference channel $m_{0}$. The probability of this event can be upper bounded as

$$
\begin{aligned}
& \mathbb{P}^{! o}\left\{m_{j}=m_{0} \mid Y_{j} \text { is transmitter in } b(0, t)\right\} \\
& =\mathbb{P}^{! o}\left\{\left|C_{j}\right| \geq M\right\} \mathbb{P}^{! o}\left\{m_{j}=m_{0}|| C_{j} \mid \geq M\right\} \\
& \quad+\mathbb{P}^{! o}\left\{\left|C_{j}\right|<M\right\} \underbrace{\mathbb{P}^{! o}\left\{m_{j}=m_{0}|| C_{j} \mid<M\right\}}_{=0} \\
& <\mathbb{P}^{! o}\left\{m_{j}=m_{0}|| C_{j} \mid \geq M\right\}=\frac{1}{M},
\end{aligned}
$$

where the last equation is explained by the fact that no particular channel should be favored after averaging over all possible point patterns, while letting $Y_{j}, X_{0}^{\mathrm{rx}}$ and $X_{0}$ fix.

The conditional expectation from (A.1) can hence be written as

$$
\begin{aligned}
\mathbb{E}^{! o} & \left\{\sum_{i} \mathbb{1}_{\left\{X_{i} \in \tilde{\Phi}_{0}(b(0, t))\right\}}|| C_{0} \mid=k\right\} \\
= & \mathbb{E}^{! o}\left\{\sum_{j=1}^{k-M+1} \mathbb{1}_{\left\{Y_{j} \text { interferes with } X_{0}^{\mathrm{rx}}\right\}}\right\} \\
= & \sum_{j=1}^{k-M+1} \mathbb{P}^{! o}\left\{Y_{j} \text { interferes with } X_{0}^{\mathrm{rx}}\right\}
\end{aligned}
$$

$$
\begin{aligned}
= & \sum_{j=1}^{k-M+1} \mathbb{P}^{! o}\left\{m_{j}=m_{0} \mid Y_{j} \text { is transmitter in } b(0, t)\right\} \\
& \times \mathbb{P}\left\{Y_{j} \in b(0, t) \mid Y_{j} \text { is transmitter }\right\} \mathbb{P}\left\{Y_{j} \text { is transmitter }\right\} \\
< & \sum_{j=1}^{k-M+1} \frac{t^{2}}{2 r^{2} M}=\frac{t^{2}(k-M+1)}{2 r^{2} M} .
\end{aligned}
$$

Substituting (A.12) and (A.10) in (A.1) yields the result for $t \leq r$.

Bound for $t>r$ : A simple upper bound is constructed by assuming that all interferers within the annulus $\lambda \pi\left(t^{2}-r^{2}\right)$ transmit in the reference channel $m_{0}$. For $t>t_{0}$, the intensity measure $\frac{\lambda}{M} \pi t^{2}$ of the PPP gives an upper bound.

\section{REFERENCES}

[1] J. Andrews, S. Shakkotai, R. Heath, N. Jindal, M. Haenggi, R. Berry, D. Guo, M. Neely, S. Weber, S. Jafar, and A. Yener, "Rethinking information theory for mobile ad hoc networks," Communications Magazine, IEEE, Dec 2008.

[2] J. Andrews, R. Ganti, M. Haenggi, N. Jindal, and S. Weber, "A primer on spatial modeling and analysis in wireless networks," Communications Magazine, IEEE, vol. 48, no. 11, pp. 156 -163, 2010.

[3] M. Haenggi, "On distances in uniformly random networks," IEEE Transactions on Information Theory, Oct 2005.

[4] F. Baccelli and B. Blaszczyszyn, "Stochastic geometry and wireless networks, volume 1+1: Theory and applications," Foundations and Trends in Networking, vol. 3, no. 3-4, pp. 249-449, 2009.

[5] S. Vanka and M. Haenggi, "Coordinated Packet Transmission in Random Wireless Networks," in IEEE Global Communications Conference (GLOBECOM'10), Miami, FL, Dec. 2010, available at http://www.nd.edu/ mhaenggi/pubs/globecom10b.pdf.

[6] P. Bermolen and F. Baccelli, "Multiple access mechanisms with performance guarantees for ad-hoc networks," in Sensor Mesh and Ad Hoc Communications and Networks (SECON), 2010 7th Annual IEEE Communications Society Conference on, 2010, pp. 1 -9.

[7] V. Nguyen Tien and F. Baccelli, "Stochastic Modeling of Carrier Sensing Based Cognitive Radio Networks," in WiOpt'10: Modeling and Optimization in Mobile, Ad Hoc, and Wireless Networks, Avignon France, 2010, pp. 594-601.

[8] J. Elsner, R. Tanbourgi, and F. Jondral, "On the Transmission Capacity of Wireless Multi-Channel Ad Hoc Networks with local FDMA scheduling," in International Congress on Ultra Modern Telecommunications and Control Systems, Oct 2010.

[9] R. Tanbourgi, J. Elsner, and F. Jondral, "Lowering Outage Probability in Ad Hoc Networks by Nearest Neighbor FDMA Scheduling," in IEEE WCNC 2011, Cancun, Mexico, Mar 2011, accepted.

[10] R. Ganti and J. Andrews, "A new method for computing the transmission capacity of non-poisson wireless networks," in Information Theory Proceedings (ISIT), 2010 IEEE International Symposium on, 2010, pp. $1693-1697$.

[11] D. Stoyan, W. Kendall, and J. Mecke, Stochastic geometry and its applications, 2nd ed. Wiley, 1995.

[12] R. Brooks, "On colouring the nodes of a network," Mathematical Proceedings of the Cambridge Philosophical Society, Apr 1941, doi:10.1017/S030500410002168X.

[13] D. J. Leith and P. Clifford, "Convergence of distributed learning algorithms for optimal wireless channel allocation," in in Proceedings of IEEE Conference on Decision and Control, 2006, pp. 2980-2985.

[14] S. Weber, J. Andrews, and N. Jindal, "An overview of the transmission capacity of wireless networks," IEEE Transactions on Communications, Dec 2010.

[15] J. Venkataraman, M. Haenggi, and O. Collins, "Shot noise models for outage and throughput analyses in wireless ad hoc networks," in Military Communications Conference, Oct 2006.

[16] I. S. Gradshteyn, I. M. Ryzik, and A. Jeffrey, Eds., Table of integrals, series, and products, 6th ed. San Diego: Acad. Press, 2000.

[17] J. Andrews, S. Weber, and M. Haenggi, "Ad hoc networks: To spread or not to spread," Communications Magazine, IEEE, Dec 2007. 